\title{
Compound K Rich Fractions Regulate NF- $\kappa B$-dependent Inflammatory Responses and Protect Mice from Endotoxin-induced Lethal Shock
}

\author{
Chul-Su Yang***, Jae-Min Yuk***, Sung-Ryong Ko***, Byung-Goo Cho***, Hyun-Joo Sohn ${ }^{* * *}$, \\ Young-Sook Kim***, Jae-Joon Wee***, Jae-Ho Do**** and Eun-Kyeong Jo****,\# \\ *Department of Microbiology and Infection Signaling Network Research Center \\ **College of Medicine, Chungnam National University, Daejeon 301-747, S. Korea \\ ***Ginseng Research Group, KT\&G Central Research Institute, 302 Shinseong-dong, Yuseong-gu, Daejeon 305-805, S. Korea
}

(Received September 18, 2008, Accepted December 11, 2008)

\begin{abstract}
In the previous studies, we isolated the compound $\mathrm{K}$ rich fractions (CKRF) and showed that CKRF inhibited Toll-like receptor (TLR) 4- or TLR9-induced inflammatory signaling. To extend our previous studies, ${ }^{1)}$ we investigated the molecular mechanisms of CKRF in the TLR4-associated signaling via nuclear factor (NF)- $\mathrm{KB}$, and in vivo role of CKRF for induction of tolerance in lipopolysaccharide (LPS)-induced septic shock. In murine bone marrow-dervied macrophages, CKRF significantly inhibited the induction of mRNA expression of proinflammatory mediators such as tumor necrosis factor- $\alpha$, interleukin-6, cyclooxygenase-2, and inducible nitric oxide synthase. In addition, CKRF significantly

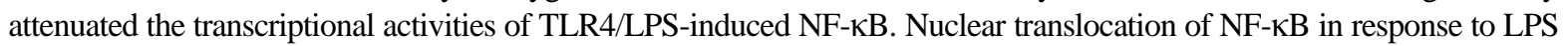
stimulation was significantly abrogated by pre-treatment with CKRF. Furthermore, CKRF inhibited the recruitment of p65 to the interferon-sensitive response element flanking region in response to LPS. Finally, oral administration of CKRF significantly protected mice from Gram-negative bacterial LPS-induced lethal shock and inhibited systemic inflammatory cytokine levels. Together, these results demonstrate that CKRF modulates the TLR4-dependent NF- $\mathrm{BB}$ activation, and suggest a therapeutic role for Gram-negative septic shock.
\end{abstract}

Key words : Compound $\mathrm{K}$ rich fractions, Toll-like receptor 4 , nuclear factor $\kappa \mathrm{B}$, endotoxemia

\section{INTRODUCTION}

The innate immune system is the first line of immune defence against invading pathogenic microbes. ${ }^{2)}$ When controlled, the innate immune responses have the power to eliminate infections. ${ }^{3)}$ Toll-like receptors (TLRs) have been widely studied and are able to recognize microbial components during the innate immune responses. ${ }^{4}$ Together with other pattern-recognition receptors, TLRs are innate receptors which play a pivotal role in sensing a variety of invading pathogens and microbes. Although TLRs mediate key immune activation upon the recognition of pathogens, hyper-activation or dysregulation of TLR signaling is linked with a number of disease conditions. Therefore, targeting TLR signaling cascades could lead to novel therapeutic strategies in the treatment of infectious and inflammatory diseases. ${ }^{2)}$

\footnotetext{
\# To whom correspondence should be addressed.

(Tel) +82-42-580-8243; (Fax) +82-42-585-3686

(E-mail) hayoungj@cnu.ac.kr
}

Among TLRs, TLR4 is known to activate the immune system by lipopolysaccharide (LPS), which is one of the most powerful immunostimulators known. Therefore TLR4 is not only involved in the induction of innate immune responses but also for the dangerous endotoxic shock, a severe inflammatory disease leading to multiorgan failure and death. ${ }^{5)}$ TLR4 can signal via two distinct sets of adaptor proteins, Mal (MyD88-adapter-like), and MyD88, and TRAM [Toll-IL-1-resistance (TIR) domaincontaining adaptor-inducing IFN- $\beta$ (TRIF)-related adaptor molecule] and TRIF. ${ }^{6,7)}$ Then, adapter Mal is required for rapid activation of the nuclear factor- $\mathrm{\kappa B}(\mathrm{NF}-\mathrm{\kappa B})$ transcription factor and the production of pro-inflammatory cytokines such as tumor necrosis factor (TNF)- $\alpha$ and interleukin (IL)-12." ${ }^{6}$ Transcription factor NF-KB is known to be activated in response to various infectious and inflammatory stimuli, and regulate the expression of a variety of genes involved in immune responses and inflammation. ${ }^{8)}$

Significant efforts have been made to discover therapeutic modalities that can control systemic inflammation 
and improve survival in septic hosts. ${ }^{9,10)}$ Ginseng, the root of Panax ginseng C. A. Meyer (Araliaceae), is one of the most widely used Chinese herbs with various pharmacological actions for a long times, however, the underlying mechanisms have not been largely understood. Ginsenoside is the major active ingredient of ginseng, and is one of the best known natural products with anti-inflammatory and anti-oxidative effects. ${ }^{11,12)}$ Ginsenoside compound $\mathrm{K}$ (CK; (20-O- $\beta$-D-glucopyranosyl-20(S)-protopanaxadiol) is speculated to be produced through fermentation of protopanaxadiol-type ginsenosides from the intestine. ${ }^{13)}$ In addition, CK was reported to be produced via transformation of ginsenosides using commercial enzyme preparations. ${ }^{14)}$

Ginsenoside metabolite CK is a promising natural product that could be applied for various pharmaceutical purposes. Several reports have shown the effects of CK on the anti-metastatic and anti-allergic activities, ${ }^{15,16)}$ apoptosis, ${ }^{17)}$ regulation of plasma glucose levels, ${ }^{18)}$ etc. Our recent studies have demonstrated a novel therapeutic role for $\mathrm{CK}$ in the treatment of lethal sepsis through the modulation of TLR4-associated signaling via glucocorticoid receptor (GR) binding. ${ }^{10)}$ In another studies from our laboratories, we isolated the $\mathrm{CK}$ rich fractions (CKRF) and examined the effects of CKRF on the inflammatory signaling during TLR-induced cellular activation. ${ }^{1)}$ In those studies, we found that CKRF specifically modulated TLR4- or TLR9-induced inflammatory signaling. ${ }^{1)}$ These findings led us to hypothesize whether CKRF regulate the systemic inflammatory responses, for in vivo roles for potential therapeutic uses, as similar to those by CK demonstrated by our previous studies. ${ }^{10)}$

In this study, we describe a novel function for CKRF in the treatment of lethal sepsis through the modulation of TLR4-associated signaling via NF-кB. We demonstrate the functional significance of $\mathrm{CKRF}$ on the regulation of TLR4-dependent signaling pathways through NF- $\kappa \mathrm{B}$ activation, and in vivo therapeutic effects against systemic inflammatory responses induced by endotoxin administration.

\section{MATERIALS AND METHOD}

\section{Isolation of compound $K$ rich fractions (CKRF)} from red ginseng extracts

CKRF was made as described previously. ${ }^{1)}$ Briefly, $100 \mathrm{~g}$ of alcohol extracts of red ginseng were incubated with $20 \mathrm{~g}$ of mixed enzymes Cellulase, Hemicellulase, Pectinase (Shin Nihon Chemical co., Japan) at $56^{\circ} \mathrm{C}$ in $10 \mathrm{~L}$ reaction medium. After incubation for 48 hours, reac- tion was terminated without any termination treatment and CKRF was collected in No2 filter paper (Whatman, USA). The CKRF in filter paper was resuspended with ethyl alcohol and centrifuged at $3000 \mathrm{rpm}$ in $10 \mathrm{~min}$. CKRF was obtain from these supernatants with vacuum evaporating under $60^{\circ} \mathrm{C}$. The yield of CKRF was 11.6 $\pm 0.1 \%$ (A dry weight basis) and the amount of CK in CKRF was $243.8 \pm 16.1 \mathrm{mg} / \mathrm{g}$. CKRF which was analyzed with HPLC. CKRF was dissolved in a mixture of dimethyl sulfoxide (DMSO; Sigma, St. Louis, MO, USA): polyethylene glycol (PEG; Sigma) 400: DDW (1:4:5) and DMSO was added to the cultures at $0.1 \%(\mathrm{v} / \mathrm{v})$ as a solvent control. The CK content was around $29 \%$ in CKRF.

\section{LPS-induced endotoxin shock and histological analysis}

All animal protocols and experiments were approved by the Institutional Animal Care and Use Committee, Chungnam National University College of Medicine (Daejeon, Korea), and complied with National Institutes of Health guidelines for the care and use of laboratory animals. All experiments described in this study were performed using C57BL/6 mice. The mice used for the lipopolysaccharide (LPS) challenge were age- and sex-matched 6-8 weeks old. Escherichia coli O26:B6 LPS (Sigma) was diluted in sterile phosphate-buffered saline (PBS) and injected into the animals intraperitoneally (i.p.).

For histopathological analysis, tissues were fixed with neutral-buffered formalin and sectioned for morphological evaluation using hematoxylin and eosin (H\&E) staining. For immunohistochemistry, the spleens were fixed by inflating the tissues and then sectioned. The slides were assessed for $C O X-2$ expression as previously described. ${ }^{9)}$

\section{Cell isolation and culture}

Bone marrow-derived macrophages (BMDMs) were obtained from 6-8 weeks old C57BL/6 female mice. Briefly, bone marrow cells from the femur and tibia were cultured for 4 days in macrophage colony-stimulating factor-containing media as described previously. ${ }^{9)}$ The murine macrophage cell line RAW264.7 was purchased from the American Type Culture Collection (TIB-71) and grown in DMEM GlutaMAX supplemented with $10 \%$ fetal bovine serum (FBS). Human embryonic kidney (HEK) 293 cells stably transfected with human TLR4, MD2, and CD14 (HEK/TLR4/MD2/CD14) was purchased from InvivoGen (San Diego, CA, USA) and grown in standard DMEM with $10 \%$ FBS supplemented with blasticidin $(10 \mu \mathrm{g} / \mathrm{ml})$ 
and Normocin $(100 \mu \mathrm{g} / \mathrm{ml})$.

\section{Reagents, DNA, and antibodies}

For in vitro experiments, ultrapure LPS (TLR4 agonist) and Dexamethasone (Dex) were obtained from InvivoGen and Sigma, respectively. DMSO was added to the cultures at $0.1 \%(\mathrm{v} / \mathrm{v})$ as a solvent control. The NF- $\mathrm{KB}$ luciferase reporter plasmid was generous gifts of Dr. Gang Min Hur (Chungnam National University, Daejeon, Korea). Antibodies against IRF3 and NF-KB p65 were obtained from Santa Cruz Biotechnology (Santa Cruz, CA, USA). AntiCOX-2 antibody was purchased from BD Transduction Laboratories (Franklin Lakes, NJ, USA).

\section{Transfection and reporter assays}

Transient transfections were performed using Lipofectamine 2000 (Invitrogen, Carlsbad, CA, USA) as per the manufacturer's instructions. The NF- $\mathrm{\kappa B}$ luciferase activity was measured using the Luciferase Assay System (Promega, Madison, WI, USA) as described previously. ${ }^{1)}$

\section{Reverse transcriptase-Polymerase chain reaction} (RT-PCR) and chromatin immunoprecipitation (ChIP) assay

RNA was extracted from the cells using TRIzol (Invitrogen). Complementary DNA was reverse transcribed from $2 \mu \mathrm{g}$ of total RNA using SuperScript II reverse transcriptase (Invitrogen) and oligo-dT-3 primer in a total volume of $20 \mu \mathrm{l}$. Using PCR, $2 \mu \mathrm{l}$ of cDNA was amplified. The primers and PCR conditions were as described previously. ${ }^{9)}$ The PCR products were resolved on $1.5 \%$ agarose gels and were stained with ethidium bromide. ChIP assays were performed as described. ${ }^{1)}$ The interferon-sensitive response element (ISRE) flanking region was amplified by PCR with the primers 5'-ATGGTCTGGGACTTTCGAGGTT-3' and 5'-TCAGGGCCCGAAAGCAAAACCA-3'.

\section{Immunostaining}

NF-KB p65 nuclear translocation in BMDMs was assessed by immunofluorescence microscopy. Cells were fixed on coverslips in $4 \%(\mathrm{w} / \mathrm{v})$ paraformaldehyde in PBS, followed by a 5 -min permeabilization in $0.25 \%$ (v/v) Triton $\mathrm{X}-100$ in PBS at $25^{\circ} \mathrm{C}$. NF- $\mathrm{\kappa B}$ p65 was detected by incubation with a 1:100 dilution of the primary $\mathrm{Ab}$ for $1 \mathrm{~h}$ at $25^{\circ} \mathrm{C}$, washing, and incubation with a 1:100 dilution of rabbit immunoglobulin G-Alexa Fluor 488 (Molecular Probes, Eugene, OR, USA) for $1 \mathrm{~h}$. Nuclei were visualized upon a $15-\mathrm{min}$ incubation with $20 \mu \mathrm{g} / \mathrm{ml}$ propidium iodide (PI). Slides were examined with a laser-scanning confocal microscope (model LSM 510; Zeiss, Oberkochen, Germany).

\section{Statistical analysis}

For parametric data, the results are expressed as the mean \pm standard error of the mean (SE), and comparisons were made using the two-tailed Student's $t$-test for paired samples. For nonparametric data, the results are expressed as the median \pm quartiles, and comparisons were made using Wilcoxon's signed-ranks test. Where indicated, an adjusted Bonferroni correction for multiple comparisons was used to reach an overall $P$ value of $<0.05$.

\section{RESULTS}

1. CKRF modulate expression of proinflammatory cytokines, cyclooxygenase (COX)-2 and inducible nitric oxide synthase (iNOS) by macrophages in response to LPS.

We recently reported that CKRF are regulated TLR4/ LPS-induced inflammatory responses in macrophages. ${ }^{10)}$ As shown in Fig. 1A, pre-treatment of BMDMs with CKRF resulted in specifically inhibited LPS-induced TNF$\alpha$ and IL-6 mRNA expression in a concentration-dependent manner, in agreement with previous report. ${ }^{10)}$ However, there was no significant changes in LPS-induced IL10 mRNA expression by pre-treated with CKRF and those that were not (Fig. 1B). We further examined whether the effect of CKRF on LPS-induced proinflammatory mediators expression. As shown in Fig. 1B, pre-treatment with CKRF significantly attenuated LPS-induced COX-2 and iNOS mRNA expression in BMDMs, in a concentrationdependent manner. Cytokine mRNA expression peaked at $4 \mathrm{~h}$ post-LPS stimulation (data not shown). These results indicate that CKRF profoundly abrogated in LPS-induced pro-inflammatory signal activation in murine macrophages.

\section{CKRF regulates NF- $\kappa B$ signaling by macrophages in response to LPS.}

$\mathrm{NF}-\mathrm{KB}$ transcription factors are rapidly activated in response to various stimuli, including cytokines and infectious agents, and regulate the expression of a variety of genes involved in immune responses and inflammation., $\left.{ }^{9}, 10\right)$ TLR stimulation results in activation of different intracellular signaling cascades, generally resulting in the activation of NF- $\mathrm{KB}$ and activating protein (AP)-1 in MyD88dependent pathways. ${ }^{19,20)}$ Therefore, we next investigated the role of CKRF in the regulation of TLR4/LPS-induced $\mathrm{NF}-\kappa \mathrm{B}$ activation. We examined the effects of CKRF on 
A

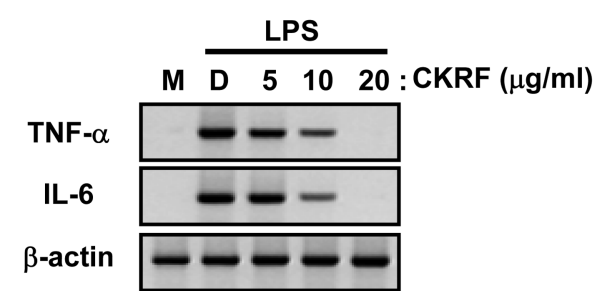

B

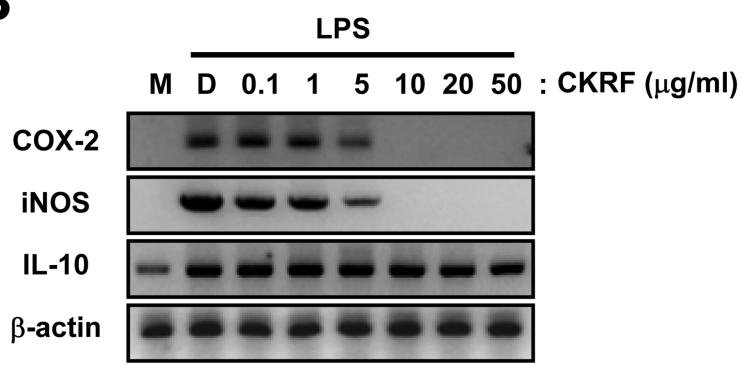

Fig. 1. Regulatory effect of CKRF on the TLR4/LPS-induced expression of proinflammatory mediators in murine macrophages. (A, B) Murine BMDMs were treated with increasing concentrations of CKRF or a solvent control for $45 \mathrm{~min}$ before LPS stimulation $(1 \mu \mathrm{g} / \mathrm{ml})$. The cells were harvested after $4 \mathrm{~h}$, total RNA was purified and semiquantitative RT-PCR analysis of TNF- $\alpha$, IL-6, IL10, COX-2 and iNOS mRNA was performed. The data are representative of three separate experiments. D, solvent control ( $0.1 \%$ DMSO); M, media control.

the modulation of NF- $\mathrm{kB}$ - and AP-1-luciferase gene activity in HEK/TLR4/MD2/CD14 cells after stimulation with LPS. Pre-treatment with CKRF significantly attenuated the transcriptional activities of NF-KB and the AP-1 in a concentration-dependent manner (Fig. 2A and data not shown). Furthermore, pre-treatment with CKRF significantly abolished the translocation of NF-KB p65 into the nucleus of BMDMs after LPS stimulation, thus confirming the role of CKRF in the modulation of LPS-mediated NF- $\mathrm{\kappa B}$ activation (Fig. 2B). However, no inhibitory effects on TLR3/poly I:C-mediated the translocation of IRF3 into the nucleus were observed upon pretreatment with CKRF (data not shown). Taken together, these data suggest that CKRF profoundly abrogated the activation of NF- $\mathrm{kB}$ signaling through MyD88-dependent pathways, but not TRIF-dependent pathways in response to LPS

3. CKRF inhibits LPS-induced p65 recruitment to the interferon-sensitive response element (ISRE) flanking region

We next examined the molecular mechanism by which CKRF specifically modulates the response to LPS. Recent studies reported that GR specially inhibits the interaction of p65 with interferon regulatory factor (IRF) complexes mediate TLR4/LPS-sepecific transcriptional responses, but not TLR3-dependent. ${ }^{10,21)}$ Therefore, we asked whether p65/IRF3 complexes mediate TLR4/LPS signaling inhibited of transcriptional responses. To examine this, we performed chromatin immunoprecipitation assays (ChIP) analysis in RAW264.7 cells (Fig. 3).

As shown in Fig. 3, both IRF3 and p65 were interacted to the promoter regions of ISRE flanking region in response to LPS, confirming the previous findings of specific recruitment of p65 to the ISRE-containing promoter in response to LPS. ${ }^{10)}$ Significantly, treatment with either CKRF or Dex attenuated the interaction of p65 to the ISRE in response to TLR4/LPS. However, in response to the TLR3 ligand, both p65 and IRF3 recruitment to the promoter regions of ISRE flanking region was not affected by treatment with either CKRF or Dex (data not shown). These data indicated that CKRF significantly inhibits the interaction of p65 with IRF3 in response to TLR4, but not TLR3, stimulation.

\section{CKRF protected mice from LPS-induced lethal shock}

We used murine sepsis model, as previously described. ${ }^{9,10)}$ Oral administration of CKRF $24 \mathrm{~h}$ before endotoxemia and then injected (i.p.) with LPS ( $40 \mathrm{mg} / \mathrm{kg}$ body weight) in C57B/L6 mice, and survival was monitored for 5 days. Although $40 \mathrm{mg} / \mathrm{kg}$ of LPS killed $78 \%$ of the control mice within 5 days postinjection, oral administration of CKRF prevented the death of the LPS-injected mice in a concentration-dependent manner. At the highest dose $(100 \mathrm{mg} /$ $\mathrm{kg}$ ) of pre-treated with CKRF, $90 \%$ of the mice were still alive, and almost $78 \%$ with $75 \mathrm{mg} / \mathrm{kg}$ of pre-treated with CKRF by day 5 post-injection of LPS ( $P<0.001$; Fig. 4$)$.

We further examined histopathological evaluation of cellular infiltration and organ injury (Fig. 5). Paraffinembedded sections were prepared from the liver and spleen of mice injected with vehicle or LPS for $24 \mathrm{~h}$ in the presence or absence of CKRF $(75 \mathrm{mg} / \mathrm{kg})$, and subjected to $\mathrm{H} \& \mathrm{E}$ staining. Our histopathological results show that bacterial lipopolysaccharide injection produced inflammatory changes (Fig. 5A), including acute inflammatory cell infiltration, congestion and marked germinal center reactions in the spleen. In the liver, acute inflammatory cell infiltrates were seen in the central vein surrounded by hepatocytes and sinusoids, and there was focal hepatocellular necrosis, Kupffer cell reactive hyperplasia and haemorrhage as well as a congested central vein. In the CKRF- 

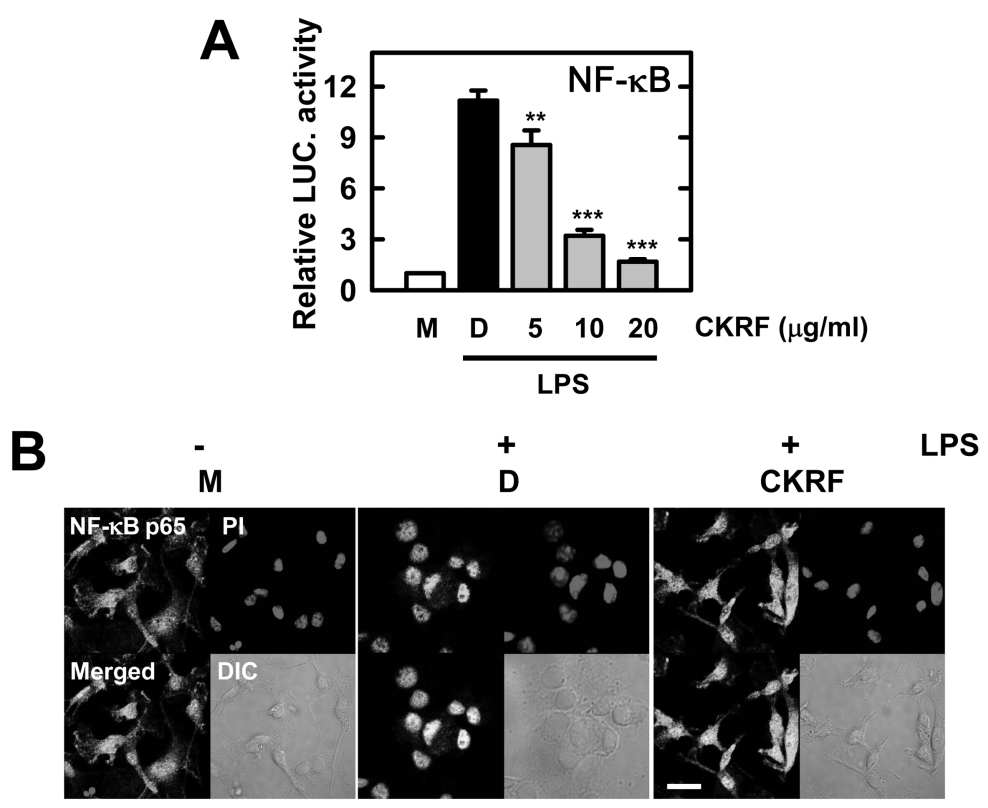

Fig. 2. Regulatory effect of CKRF on the TLR4/LPS-induced NF- $\mathrm{KB}$ signaling in murine macrophages. (A) hTLR4-HEK293 cells were transiently transfected with pNF-KB-luc. At $24 \mathrm{~h}$ post-transfection, the cells were stimulated for $4 \mathrm{~h}$ with LPS $(1 \mu \mathrm{g} / \mathrm{ml}) \mathrm{in}$ the presence or absence of CKRF at the indicated concentrations. The cells were then harvested, and luciferase activity was measured and corrected for differences in transfection efficiency based on the $\beta$-galactosidase activity. The luciferase activities shown are the mean $\pm \mathrm{SD}$ of three independent experiments. Significant differences from the control values (without CKRF pretreatment $)$ are indicated as $* *(P<0.01)$ or $* * *(P<0.001)$. (B) Murine BMDMs were stimulated with LPS $(1 \mu \mathrm{g} / \mathrm{ml})$ in the presence or absence of CKRF $(10 \mu \mathrm{g} / \mathrm{ml})$ and harvested $4 \mathrm{~h}$ later for immunofluorescence microscopy. The cells were fixed and stained with NF- $\mathrm{KB} \mathrm{Ab}$ and PI, followed by examination under a confocal microscope (model LSM 510; Zeiss, Oberkochen, Germany). The data are representative of three separate experiments. Scale bar: $100 \mu \mathrm{m}$. D, solvent control (0.1\% DMSO); M, media control.

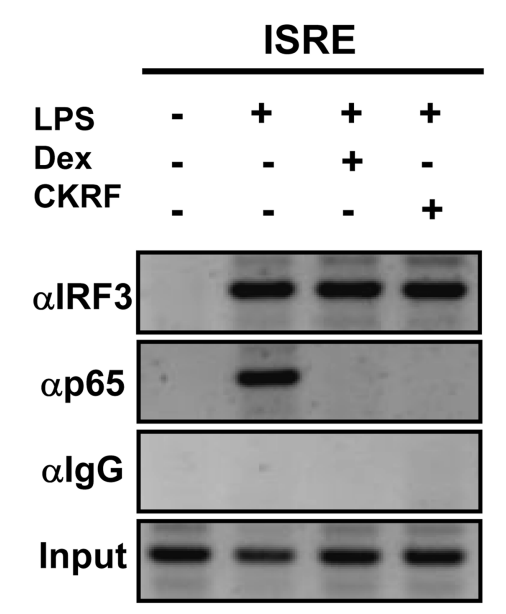

Fig. 3. p65 recruitment to the proximal promoter region of ISRE is specifically induced by LPS and inhibited by CKRF. RAW264.7 cells were treated with LPS $(1 \mu \mathrm{g} / \mathrm{ml})$, Dex $(10 \mu \mathrm{g} / \mathrm{ml})$ or CKRF $(10 \mu \mathrm{g} / \mathrm{ml})$ for $1 \mathrm{~h}$. ChIP assays were performed using antibodies against IRF3, p65, or IgG. Immunoprecipitated DNA was analyzed by PCR using primers specific for the promoter. A representative experiment of three independent replicates with similar results is shown.

treated mice, much less damage was seen than in the control mice after injection with LPS (Fig. 5A). When the
COX-2 expression were compared in spleens from control and CKRF-treated mice with LPS injection, the COX-2 


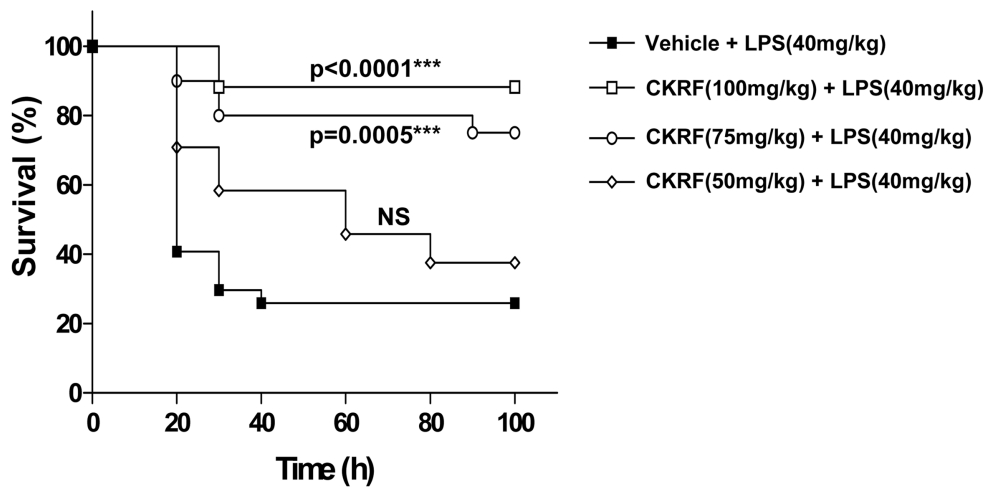

Fig. 4. CKRF treatment prevents lethal endotoxemia. (A) Mice ( $\mathrm{n}=25$ per group) received either CKRF (50, 75, or $100 \mathrm{mg} / \mathrm{kg}$; administered orally) or vehicle only at $24 \mathrm{~h}$ before a lethal dose of endotoxin (40 mg LPS/kg; intraperitoneal injection). Survival was recorded for the mice subjected to endotoxemia. Viability was assessed every $5 \mathrm{~h}$ for the first $40 \mathrm{~h}$ and every $10 \mathrm{~h}$ thereafter. There was no further increase in death after $90 \mathrm{~h}$. Statistical differences $(* * *, P<0.001)$ compared to the control mice are indicated. NS, non-specific.

A

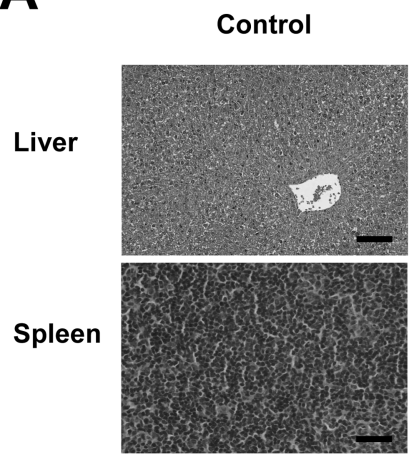

B

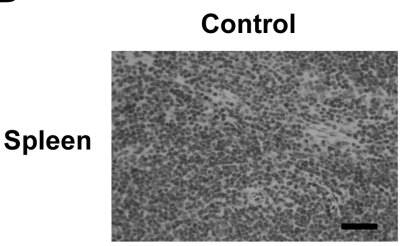

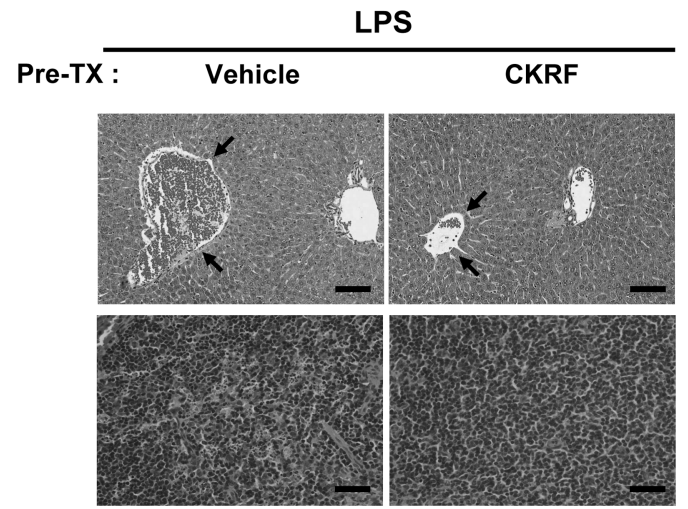

LPS

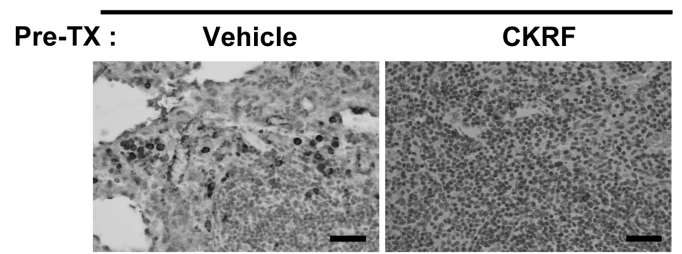

Fig. 5. Histopathological examination and immunohistochemistry analysis showed CKRF treatment prevents LPS-induced lethal shock. (A) Sections of liver (top) and spleen (bottom) from mice subjected to endotoxemia $24 \mathrm{~h}$ after oral administration of either CKRF $(75 \mathrm{mg} / \mathrm{kg}$ ) or vehicle. Black arrows indicate a congested central vein and hemorrhage in liver. H\&E staining was performed (scale bars: upper, $50 \mu \mathrm{m}$; lower, $200 \mu \mathrm{m}$ ). (B) COX-2 immunoreactivity was compared in mice subjected to endotoxemia $24 \mathrm{~h}$ after oral administration of either CKRF $(75 \mathrm{mg} / \mathrm{kg})$ or vehicle. Spleen sections were stained with anti-COX2 antibody (scale bar: $100 \mu \mathrm{m}$ ). The left figure is representative of the normal control mice. The middle and right figures show the liver and spleen injected with LPS. The images are representative of sections from five mice per group.

expression of CKRF-treated mice was found to be significantly lower than that of control mice (Fig. 5B). The pathological changes caused by LPS were significantly alleviated in CKRF-treated mice in all two organs. These data thus support a strong in vivo activity of CKRF for prevention of endotoxin-induced lethal shock.

\section{DISCUSSION}

Triggering of TLR signaling can lead to the production of inflammatory mediators, which promote the elimination of infectious agents. The ability to orchestrate biological activities of TLR agonists often results in diverse host responses to influence the outcome of the inflammatory 
responses. ${ }^{22)}$ Excessive inflammation during microbial infection results in a marked tissue damage and lethal septic shock. ${ }^{8)}$ The mortality rate of sepsis may range above $30 \%$ in the patients with severe cases. ${ }^{23)}$ Although much effort is being contributed to critical care, there is no specific, efficient therapy applicable to patients with septic shock. ${ }^{24)}$ Full activation of multiple TLR signalling pathways may lead to hyper-inflammation and organ injury during sepsis, although many aspects of immunopathogenesis in sepsis patients need to be further elucidated. ${ }^{25}$ )

Ginsenoside metabolite $\mathrm{CK}$ is a promising natural product that could be applied for the pharmaceutical purpose or treatment of various pathologic conditions. Previous studies demonstrated that anti-metastatic and anti-allergic activities by the ginsenosides were mediated by $\mathrm{CK} .{ }^{15,16)}$ Our recent findings revealed that $\mathrm{CK}$ attenuated proinflammatory cytokine production by macrophages and significantly increased the survival rate of septic mice. ${ }^{10)}$ However, in those studies, we found that CK can serve as an agonist ligand for GR and regulate the MyD88dependent signaling via GR engagement. ${ }^{10)}$ Currently available steroidal drugs generated enhanced binding to the GR could produce important and serious side effects. ${ }^{26,27)}$ Therefore, new compounds with reduced activities through GR engagement, and increased action on the anti-inflammatory effects should be desirable for both clinical applications and basic research.

In the current study, CKRF was isolated from Korean red ginseng ${ }^{1}$. Red ginseng was reported to contain higher amounts of protopanaxadiol-type ginsenosides (Rb1, Rb2, $\mathrm{Rc}, \mathrm{Rd}, \mathrm{Rg} 3$ ) than white ginseng. ${ }^{28)}$ Recent studies have reported that the protopanaxadiol-type ginsenosides are highly associated with anti-inflammatory responses in various types of cells. ${ }^{29-31)}$ Therefore we were to investigate the effects of CKRF including a variety of protopanaxadiol-type ginsenosides, which could contribute to develop effective anti-inflammatory agents containing reduced amounts of purified CK. As CKRF contained around $29 \%$ of CK in their contents, CKRF significantly regulate inflammatory signaling during TLR4- or TLR9induced cellular activation, ${ }^{1)}$ whereas CK significantly inhibits inflammatory signalling during TLR2- or TLR4mediated activation. ${ }^{10)}$ Therefore it may be useful as a therapeutic tool for the potential treatment of sepsis. In this study, we focused on the efficacy of CKRF using in vivo model of sepsis. We found that CKRF treatment effectively reduced organ inflammation and damage following injection with LPS, and $C O X-2$ expression in the spleens, compared with those that received LPS but not
CKRF. These data correlated with our recent findings with $\mathrm{CK},{ }^{10)}$ and further demonstrate that CKRF can be used as a therapeutic purpose for endotoxin-induced lethal shock in vivo.

Nevertheless, these studies demonstrate that negative regulation of inflammatory responses induced by CKRF is thought to result, at least in part, from the ability of GR to interfere with the activities of NF- $\mathrm{KB}$ via transrepression. ${ }^{10,32)}$ Our data showed that CKRF suppresses TLR4/ LPS-induced inflammatory gene expression by inhibiting the recruitment of p65 to the ISRE in response to LPS (see Fig. 3). Previously, it was demonstrated that GR effectively disrupted the formation of an IRF3/p65 activator/coactivator complex required for the activation of ISRE-containing promoters in TLR4/LPS signalling. ${ }^{21)}$ These findings may indicate that the binding of CK or other ginsenosides of CKRF to GR disrupts an IRF3/p65 complex required for the activation of ISRE-containing promoters by TLR4/LPS-dependent signaling. Further studies should clarify which components of CKRF modulate the GR-mediated transrepression.

Pretreatment with CKRF significantly inhibited the mRNA expression of TNF- $\alpha$, IL-6, COX-2, and iNOS by BMDMs in response to LPS. As CKRF did not modulate the expression of IL-10, the suppressive effects of CKRF on the inflammatory mediator gene expression might be not due to an increase of anti-inflammatory responses. These data partly agree with our previous studies that CK substantially inhibited the secretion of inflammatory cytokines and activation of MAPK and NF- $\kappa B$ in BMDMs in response to TLR2/BLP or TLR4/LPS, but not TLR3/poly I:C. ${ }^{10)}$ We also showed that CKRF affected the NF- $\mathrm{KB}$ signaling pathways initiated by TLR4/LPS. Of note, CKRF inhibited the transcription of NF-KB-luciferase in HEK/TLR4/MD2/CD14, and translocation of NF- $\mathrm{KB}$ in BMDMs, when stimulated with LPS. In supporting the important role of NF- $\mathrm{kB}$ activity on systemic inflammation, Ye et al. have recently reported that endothelial NF$\kappa \mathrm{B}$ activity is critically required for the inflammatory and injurious responses leading to septic multiple organ inflammation and injury. ${ }^{33)}$

$\mathrm{NF}-\kappa \mathrm{B}$ activation is a key component of host immune response, but also drive to the progression of systemic inflammation and septic pathology. ${ }^{34)}$ The pleiotropic transcription factor NF-KB is a central downstream element of TLR-dependent signaling and plays an important role in the regulation of multiple physiologic and pathologic phenomena, including apoptosis, cell growth, stress response, innate immunity and septic shock. ${ }^{35)}$ In addition, patients 
with septic shock showed an increased NF- $\kappa \mathrm{B}$ activity, which may help to predict the outcome of sepsis, as a potential early prognostic marker for severe sepsis. ${ }^{36)}$ Moreover, specific blockade of NF- $\mathrm{KB}$ with peptide containing the NEMO-binding domain inhibited the LPSdelayed neutrophil apoptosis, ${ }^{37)}$ which might affect the resolution of inflammation. ${ }^{38)}$ A detailed understanding of the molecular basis of the regulation of NF- $\kappa B$ activation by CKRF is needed in order to specifically block inflammatory signaling during sepsis or severe inflammation, while avoiding adverse effects induced by GR stimulation. Future studies should reveal the precise components and molecular mechanisms by which CKRF regulates the individual TLR-induced inflammatory signaling pathways.

In summary, this study identified CKRF function as immunomodulatory factors with the capacity to inhibit or attenuate the TLR4/LPS-dependent inflammatory responses in vitro and in vivo. Our data strongly suggest a potential clinical utility of CKRF in the treatment of sepsis or severe inflammation through the modulation of excessive inflammatory responses.

\section{ACKNOWLEDGMENTS}

We thank Drs. Shengjin Li and Jin-Man Kim (Chungnam National University) for technical supports. This research was supported by the Korea Science \& Engineering Foundation through the Infection Signaling Network Research Center (R13-2007-020-01000-0) at Chungnam National University, KT\&G Central Research Institute (2006), and the Korean Society of Ginseng (2005).

\section{REFERENCES}

1. Yang, C. S., Ko, S. R., Cho, B. G., Lee, J. Y., Kim, K. H., Shin, D. M., Yuk, J. M., Sohn, H. J., Kim, Y. S., Wee, J. J., Do, J. H. and Jo, E. K. : Compound K (CK) Rich Fractions from Korean Red Ginseng Inhibit Toll-like Receptor (TLR) 4- or TLR9-mediated Mitogen-activated Protein Kinases Activation and Pro-inflammatory Responses in Murine Macrophages. J. Ginseng Res. 31, 181-190 (2007).

2. Carpenter, S. and O'Neill, L. A. : How important are Toll-like receptors for antimicrobial responses? Cell. Microbiol. 9, 1891-901 (2007).

3. Takeda, K. and Akira, S. : Toll-like receptors in innate immunity. Int Immunol. 17, 1-14 (2005).

4. Jo, E. K., Yang, C. S., Choi, C. H. and Harding, C. V. : Intracellular signaling cascades regulating innate immune responses to Mycobacteria: branching out from Toll-like receptors.
Cell. Microbiol. 9, 1087-1098 (2007).

5. Hunter, P. : Sepsis under siege: a new understanding of sepsis might lead to the development of therapies to treat septic shock. EMBO Rep. 7, 667-669 (2006).

6. Fitzgerald, K. A., Palsson-McDermott, E. M., Bowie, A. G., Jefferies, C. A., Mansell, A. S., Brady, G., Brint, E., Dunne, A., Gray, P., Harte, M. T., McMurray, D., Smith, D. E., Sims, J. E., Bird, T. A. and O'Neill, L. A. : Mal (MyD88-adapterlike) is required for Toll-like receptor-4 signal transduction. Nature. 413, 78-83 (2001).

7. Fitzgerald, K. A., Rowe, D. C., Barnes, B. J., Caffrey, D. R., Visintin, A., Latz, E., Monks, B., Pitha, P. M. and Golenbock, D.T. : LPS-TLR4 signaling to IRF-3/7 and NF-kappaB involves the toll adapters TRAM and TRIF. J Exp Med. 198, 1043-1055 (2003).

8. Cohen, J. : The immunopathogenesis of sepsis. Nature. $\mathbf{4 2 0}$, 885-891 (2002).

9. Yang, C. S., Lee, D. S., Song, C. H., An, S. J., Li, S., Kim, J. M., Kim, C. S., Yoo, D. G., Jeon, B. H., Yang, H. Y., Lee, T. H., Lee, Z. W., El-Benna, J., Yu, D. Y. and Jo, E. K. : Roles of peroxiredoxin II in the regulation of proinflammatory responses to LPS and protection against endotoxin-induced lethal shock. J Exp Med. 204, 583-594 (2007).

10. Yang, C. S., Ko, S. R., Cho, B. G., Shin, D. M., Yuk, J. M., Li, S., Kim, J. M., Evans, R. M., Jung, J. S., Song, D. K. and Jo, E. K.: The Ginsenoside Metabolite Compound K, a Novel Agonist of Glucocorticoid Receptor, Induces Tolerance to Endotoxin-induced Lethal Shock. J. Cell. Mol. Med. 12, 1739-1753 (2008).

11. Li, Y. N., Wu, Y. L., Jia, Z. H. and Qi, J. S.: Interaction between COX-2 and iNOS aggravates vascular lesion and antagonistic effect of ginsenoside. J. Ethnopharmacol. 119, 305-311 (2008).

12. Seo, Y. J., Kwon, M. S., Choi, H. W., Jang, J. E., Lee, J. K., Sun, Y., Jung, J. S., Park, S. H. and Suh, H.W. : Intracerebroventricular ginsenosides are antinociceptive in proinflammatory cytokine-induced pain behaviors of mice. Arch Pharm. Res. 31, 364-369 (2008).

13. Bae, E. A., Kim, N. Y., Han, M. J., Choo, M. K. and Kim, D. H. : Transformation of ginsenoside to compound K (IH-901) by lactic acid bacteria of human intestine. J. Microbiol. Biotechnol. 13, 9-14 (2003).

14. Jiang, B. H., Han, Y., Zhao, Y. Q., Hu, X. M. and Zheng, L. $\mathrm{X}$. : Optimization of enzymatic translation for preparation ginsenoside compound $\mathrm{K}$ in total saponins of Panax notoginseng. Chin. Tradit. Herb Drugs. 35, 986-988 (2004).

15. Hasegawa, H., Sung, J. H. and Hur, J. D. : Ginseng intestinal bacterial metabolite IH901 as a new antimetastatic agent. Arch. Pharm. Res. 20, 539-544 (1997).

16. Bae, E. A., Choo, M. K., Park, E. K., Park, S. Y., Shin, H. Y. and Kim, D. H. : Metabolism of ginsenoside R(c) by human intestinal bacteria and its related antiallergic activity. Biol. 
Pharm. Bull. 25, 743-747 (2002).

17. Park, E. J., Zhao, Y. Z., Kim, J. and Sohn, D. H. : A ginsenoside metabolite, 20-O-beta-D-glucopyranosyl-20(S)-protopanaxadiol, triggers apoptosis in activated rat hepatic stellate cells via caspase-3 activation. Planta Med. 72, 1250-1253 (2006).

18. Yoon, S. H., Han, E. J., Sung, J. H. and Chung, S. H. : Antidiabetic effects of compound $\mathrm{K}$ versus metformin versus compound K-metformin combination therapy in diabetic $\mathrm{db} /$ db mice. Biol. Pharm. Bull. 30, 2196-2200 (2007).

19. Akira, S. and Hoshino, K. : Myeloid differentiation factor 88dependent and -independent pathways in Toll-like receptor signaling. J. Infect. Dis. 187, S356-S363 (2003).

20. Akira, S. and Takeda, K. : Toll-like receptor signaling. Nat. Rev. Immunol. 4, 499-511 (2004).

21. Ogawa, S., Lozach, J., Benner, C., Pascual, G., Tangirala, R. K., Westin, S., Hoffmann, A., Subramaniam, S., David, M., Rosenfeld, M. G. and Glass, C. K. : Molecular determinants of crosstalk between nuclear receptors and toll-like receptors. Cell. 122, 707-721 (2005).

22. Toshchakov, V., Jones, B. W., Perera, P. Y., Thomas, K., Cody, M. J., Zhang, S., Williams, B. R., Major, J., Hamilton, T. A., Fenton, M. J. and Vogel, S. N. : TLR4, but not TLR2, mediates IFN-beta-induced STAT1alpha/beta-dependent gene expression in macrophages. Nat Immunol. 3, 392-398 (2002).

23. Bernard, R., Vincent, J. L., Laterre, P. F., LaRosa, S. P., Dhainaut, J. F., Lopez-Rodriguez, A., Steingrub, J. S., Garber, G. E., Helterbrand, J. D., Ely, E. W. and Fisher, C. J. : Efficacy and safety of recombinant human activated protein $\mathrm{C}$ for severe sepsis. N. Engl. J. Med. 344, 699-709 (2001).

24. Imahara, S. D. and O'Keefe, G. E. : Genetic determinants of the inflammatory response. Curr. Opin. Crit. Care 10, 318324 (2004).

25. Weighardt, H. and Holzmann, B. : Role of Toll-like receptor responses for sepsis pathogenesis Immunobiology. 212, 715722 (2008).

26. Raynauud, P. and Ojasoo, T. : The design and use of sex-steroid antagonists, J. Steroid Biochem. 25, 811-833 (1986).

27. Bratoeff, E., Ramírez, E., Flores, E., Sánchez, M., Heuze, I. and Cabeza, M. : New aromatic esters of progesterone as antiandrogens, J. Enz. Inh. Med. Chem. 19, 99-105 (2004).

28. Li, H., Lee, J. H. and Ha, J. M. : Effective purification of ginsenosides from cultured wild ginseng roots, red ginseng, and white ginseng with macroporous resins. J. Microbiol. Biotechnol. 18, 1789-1791 (2008).

29. Joo, S. S., Yoo, Y. M., Ahn, B. W., Nam, S. Y., Kim, Y. B., Hwang, K. W. and Lee, do. I. : Prevention of inflammationmediated neurotoxicity by $\operatorname{Rg} 3$ and its role in microglial activation. Biol Pharm Bull. 31, 1392-1396 (2008).

30. Yang, Z. G., Sun, H. X. and Ye, Y. P. : Ginsenoside Rd from Panax notoginseng is cytotoxic towards HeLa cancer cells and induces apoptosis. Chem Biodivers. 3, 187-197 (2006).

31. Park, E. K., Shin, Y. W., Lee, H. U., Kim, S. S., Lee, Y. C., Lee, B. Y. and Kim, D. H. : Inhibitory effect of ginsenoside $\mathrm{Rb} 1$ and compound $\mathrm{K}$ on $\mathrm{NO}$ and prostaglandin E2 biosyntheses of RAW264.7 cells induced by lipopolysaccharide. Biol Pharm Bull. 28, 652-566 (2005).

32. De Bosscher, K., Vanden Berghe, W. and Haegeman, G. : The interplay between the glucocorticoid receptor and nuclear factor-kappaB or activator protein-1: molecular mechanisms for gene repression. Endocr. Rev. 24, 488-522 (2003).

33. Ye, X., Ding, J., Zhou, X., Chen, G. and Liu, S. F. : Divergent roles of endothelial NF-kappaB in multiple organ injury and bacterial clearance in mouse models of sepsis. J. Exd. Med. 9, 1303-1315 (2008).

34. Liu, S. F. and Malik, A. B. : NF-кB activation as a pathologic mechanism of septic shock and inflammation. Am. J. Physiol. Lung Cell. Mol. Physiol. 290, L622-L645 (2006).

35. Brown, M. A. and Jones, W. K. : NF-kappaB action in sepsis: the innate immune system and the heart. Front. Biosci. 1, 1201-1217 (2004).

36. Arnalich, F., Garcia-Palomero, E., Lopez, J., Jimenez, M., Madero, R., Renart, J., Vazquez, J. J. and Montiel, C. : Predictive value of nuclear factor kappaB activity and plasma cytokine levels in patients with sepsis. Infect. Immun. 68, 1942-1945 (2000).

37. Choi, M., Rolle, S., Wellner, M., Cardoso, M. C., Scheidereit, C., Luft, F. C. and Kettritz, R. : Inhibition of NF-kappaB by a TAT-NEMO-binding domain peptide accelerates constitutive apoptosis and abrogates LPS-delayed neutrophil apoptosis. Blood. 102, 2259-2267 (2003).

38. Savill, J. S., Wyllie, A. H., Henson, J. E., Walport, M. J., Henson, P. M. and Haslett, C. : Macrophage phagocytosis of aging neutrophils in inflammation. Programmed cell death in the neutrophil leads to its recognition by macrophages. $J$. Clin. Invest. 83, 865-875 (1989). 